\title{
Therapeutic Approaches to Opioid Use Disorder: What is the Current Standard of Care?
}

\author{
Joseph A Carley (iD \\ Tyler Oesterle (D)
}

Department of Psychiatry and Psychology, Mayo Clinic, Rochester, MN, USA

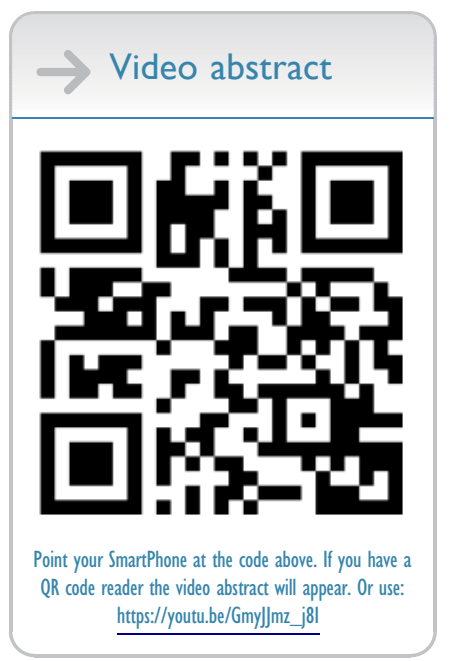

Correspondence: Joseph A Carley

Department of Psychiatry and Psychology,

Mayo Clinic, 200 Ist Street SW,

Rochester, MN, 55905, USA

Tel +l 507 284-2088

Email carley.joseph@mayo.edu

\begin{abstract}
Opioid use disorder is a frequent cause of suffering to communities worldwide. Therapeutic approaches to opioid use disorder include screening, appropriate assessment and diagnosis, consideration of level of care, acute management of overdose or withdrawal, treatment with medications, psychotherapeutic approaches, and community support. People who struggle with addiction to opioids often suffer deeply from direct and indirect consequences of use. Subsequently, it is critical that all medical providers understand the appropriate treatment options for opioid use disorder. This paper provides a comprehensive overview of the therapeutic options available for treatment of this chronic disease.
\end{abstract}

Keywords: addiction, opioid, substance use, chemical dependency, primary care, medication-assisted treatment

\section{Introduction}

Opioid use disorder (OUD) is a leading cause of suffering to communities throughout the world. Opioids include all substances which activate opioid receptors in the brain and body. Addiction-related problems can arise from both the use of illicit opioids like heroin as well as the misuse of prescribed opioid medications like morphine. OUD is a disease characterized by loss of control of use, cravings for continued use, use despite negative consequences, development of tolerance, and withdrawal symptoms. Therapeutic approaches to OUD include screening, engagement, appropriate assessment, diagnosis, consideration of level of care, acute management of overdose or withdrawal, treatment with medications, psychotherapeutic approaches, and community support. ${ }^{1-3}$

\section{Screening and Engagement}

Outside of treatment seeking or self-referred patients, initiation of treatment for OUD often depends on successful screening within primary care settings or emergency departments. There is significant stigma and shame surrounding addiction which causes many patients to avoid asking for help. Effectively engaging patients who struggle with OUD requires empathic, non-judgmental approaches. Miller and Rollnick, the founders of motivational interviewing, describe an underlying spirit or mindset of partnership, acceptance, compassion, and evocation. ${ }^{4}$ There are several helpful screening tools which can be integrated into various care settings. Routine screening for substance use disorders (SUD) in general medical settings helps to normalize addiction as a health issue. ${ }^{5}$ Screening questionnaires facilitate the first step in identifying the potential need for additional assessment to properly guide 
treatment planning. When screening for OUD, it is critical to consider co-occurring medical and psychiatric conditions. OUD frequently develops in people suffering from chronic pain, depression, anxiety, suicidal ideation, and vice versa. ${ }^{2,6}$ Depending on use practices, people who inject or insufflate opioids are at increased risk of contracting HIV/AIDS and HCV. Furthermore, people with OUD commonly have a history of hazardous use of multiple other substances. ${ }^{7}$ Screening for and treating these interrelated issues provides people with the best chance of recovery. ${ }^{8}$

\section{Diagnosis}

A diagnosis of OUD is made by taking an inventory of the symptoms and the related distress or impairment. In the US the American Psychiatric Association's Diagnostic and Statistical Manual of Mental Disorders, 5th Edition (DSM5) sets forth 11 criteria to be assessed over a 12-month period. These include using more than intended, attempts to stop using, extensive time spent using or obtaining the opioid, cravings, not meeting responsibilities due to use, relationship problems, giving up activities, use in risky situations, continued use despite emotional or physical consequences, tolerance build up, and withdrawals. DSM-5 delineates mild as having 2-3, moderate as 4-5, and severe as 6 or more criteria within 12 months. ${ }^{9}$

\section{Level of Care Assessment}

Once an OUD is identified, it is useful to assess for the appropriate level of care (ie, detox, outpatient, inpatient, medical hospitalization). The American Society of Addiction Medicine (ASAM) criteria provide a useful conceptual framework in developing efficient treatment plans for SUDs. These criteria parallel the commonly utilized biopsychosocial model. The ASAM criteria are widely used in assessing patient care needs for addiction and cooccurring disorders. There are 6 dimensions within the ASAM criteria to guide the determination of where and how to best support patients struggling with addiction. The first dimension evaluates intoxication and withdrawal potential. The second dimension considers biomedical conditions. The third dimension accounts for emotional, behavioral, or cognitive conditions. The fourth dimension assesses readiness to change. The fifth dimension looks at relapse or continued use potential. The sixth criteria takes an inventory of the individual's recovery environment. ${ }^{10}$ In the following sections, we follow the conceptual framework of the ASAM criteria to discuss treatment of OUD.

\section{Overdose Reversal}

The first step in the therapeutic approach to OUD is assessing the level of intoxication and withdrawal risk. For OUD the most immediate clinical concern is for potential overdose and respiratory suppression. If an opioid-related overdose is suspected, then naloxone is the first-line treatment. Naloxone is a life-saving medication which acts as an antagonist at the mu-opioid receptor to rapidly reverse an opioid-related overdose. Naloxone is increasingly present in various medical treatment facilities, is prescribed to patients when overdose risk is present, and is becoming more widely available to the public given its relative benign safety profile. The analogy of a fire extinguisher is often used to describe the importance of having naloxone on-hand, as one keeps naloxone readily available for emergencies but hopes never to have to use it. Naloxone is frequently administered as either an intramuscular injection or nasal spray. It is important to note that naloxone has a relatively short half-life of 30-60 minutes which may not outlast the underlying offending opioid and thus may require repeat administration. If naloxone is needed for an overdose outside of the hospital setting, then in most cases emergency medical response systems should be activated for further support. ${ }^{11-14}$

\section{Withdrawal Management}

Withdrawal from opioids is often dismissed as non-lethal and, while this is mostly accurate, this can contribute to an underappreciation of the distress involved and subsequently lead to underutilization of readily available medications. Treatment of opioid withdrawal can be accomplished either directly with opioid agonist medications or indirectly with non-opioids frequently referred to as "supportive" or "comfort" medications. Opioid agonists commonly used in the treatment of opioid withdrawal are also used in OUD treatment (ie, methadone and buprenorphine). Agonist treatment is very effective for opioid withdrawal, however, regulatory barriers to prescribing often limit their use in practice (see medication assisted treatment section of this review). Nonopioid medications target the symptoms of opioid withdrawal and are typically selected for their more favorable safety profile and lower level of regulatory or controlled status compared to opioid agonists. A standard component is clonidine, the alpha 2 adrenergic agonist, which is given to reduce the noradrenaline mediated elements of withdrawal including body aches, diaphoresis, tremors, and elevated blood pressure. Another frequently prescribed 
comfort medication for opioid withdrawal is the antidepressant trazodone, which serves as a sleep aid to address withdrawal induced insomnia. For withdrawal-related GI upset, loperamide is an option which activates enteric opioid receptors without being otherwise absorbed. Finally, people experiencing opioid withdrawal frequently have significant restlessness and physical anxiety which can be partially alleviated by the antihistamine hydroxyzine. ${ }^{15,16}$ See Table 1 for a summary of supportive medications for opioid withdrawal.

\section{Comorbid Conditions}

In further assessing the appropriate level of care for OUD, the presence and severity of medical comorbidities will influence where and when treatment can safely and effectively take place. Medically unstable patients with OUD will first require acute stabilization. Similarly, the type and degree of co-occurring mental health conditions should guide the practitioner in developing efficient care plans. Most directly, if there is active suicidal ideation then safety planning must be prioritized, and the patient may need inpatient psychiatric treatment prior to addiction treatment. Beyond acute medical and psychiatric issues, patients benefit from simultaneous management of comorbid conditions such as chronic pain, depression, and anxiety along with the opioid use disorder. ${ }^{2,6,8,17}$

\section{Readiness to Change, Relapse Potential, and Recovery Environment}

Ultimately for the care plan to be effective, the type of treatment must also be matched to the patient's level of motivation and desired treatment focus. Consideration must also be given to risk of relapse or continued use in the patient's current environment. Motivated patients with high relapse risk in their current living circumstances and/or failed attempts to quit with outpatient treatment may benefit from more intensive treatment settings such intensive outpatient programs or inpatient residential rehabilitation programs. These higher levels of care may not always be available, or the patient may not be willing to engage with them.

\section{Overview of Interventions}

The most widely available therapeutic setting for the treatment of OUD is the primary care clinic. There is growing support for general practitioner office-based management of OUD utilizing buprenorphine, also frequently referred to as office-based opioid treatment (OBOT) ${ }^{18}$ However, in parallel to this there are dedicated substance use disorder specialty practices utilizing medications for OUD commonly known as medication assisted treatment (MAT) that can assist with the more complicated patients. Of critical importance is the referral to psychotherapy for people receiving treatment MAT for OUD, especially when considering the frequency of underlying trauma, anxiety, and depression. There are multiple evidenced-based talk therapy and counseling options which should be encouraged in supporting recovery from opioid use disorders. A somewhat unique paradigm of recovery from the disease of addiction is the existence of robust community-based approaches, including peer-support meetings, therapeutic housing, and treatment "drug" courts.

\section{Medications Assisted Treatment (MAT)}

When an OUD is identified and there are plans in place for appropriately managing active intoxication or withdrawal, then consideration should be given to medication treatment.

Table I Nonopioid Withdrawal Medication Options

\begin{tabular}{|l|l|l|l|}
\hline Medication (Dosage) & Mechanism & Withdrawal Symptom & $\begin{array}{l}\text { Adverse } \\
\text { Effects }\end{array}$ \\
\hline $\begin{array}{l}\text { Clonidine (0.05mg-0.2mg PO q 4-6 } \\
\text { hours) }\end{array}$ & Alpha 2 adrenergic agonist & $\begin{array}{l}\text { Body aches, sweats, tremors, } \\
\text { elevated BP }\end{array}$ & Hypotension \\
\hline Trazodone (50-100mg PO qhs) & $\begin{array}{l}\text { Antagonist at 5HT2A, (HI) alpha I adrenergic } \\
\text { receptors }\end{array}$ & Insomnia & $\begin{array}{l}\text { Morning } \\
\text { grogginess }\end{array}$ \\
\hline $\begin{array}{l}\text { Loperamide (2-4mg PO q 4-6 hours) } \\
\text { Hydroxyzine (25-50mg PO q 4-6 } \\
\text { hours) }\end{array}$ & Anteric opioid receptor agonist & Gl upset & Constipation \\
\hline
\end{tabular}

Abbreviations: OUD, opioid use disorder; mg, milligrams; PO, oral intake; GI, gastrointestinal; q, "quaque” or “per”; qhs, bedtime; 5HT2A, (HI), serotonin receptor subtype; BP, blood pressure. 
There are three primary US FDA approved and WHO recommended medications for OUD: buprenorphine, naltrexone, and methadone. ${ }^{19}$

\section{Buprenorphine}

Buprenorphine is a partial mu opioid receptor agonist with a high binding affinity and a long duration of action. ${ }^{20}$ Studies comparing maintenance opioid agonist buprenorphine treatment to tapering and discontinuing the buprenorphine show lower rates of relapse in the buprenorphine maintenance groups. ${ }^{21}$ Moderate to severe opioid use disorder can be effectively and safely managed with buprenorphine maintenance in most cases. ${ }^{22}$ When prescribing buprenorphine, it is important to be aware of the potential for precipitated withdrawal. Precipitated withdrawal occurs when a full mu opioid receptor agonist is present and buprenorphine's high binding affinity displaces the full agonists causing a rapid drop in the mu receptor activation from full to partial. ${ }^{23}$ The key is to wait to start buprenorphine until moderate withdrawal symptoms are present. The amount of time from last use to moderate withdrawal varies depending on half-life of the opioid. The level of withdrawals can be quantified using a scale such as the clinical opioid withdrawal scale (COWS) which gives a score range of 13-24 for moderate withdrawal. ${ }^{24}$ There is evidence to support similar outcomes for unobserved "at home" inductions compared to in office inductions. ${ }^{25-29}$ However, an inoffice induction is ideal, especially for higher risk patients such as those with significant medical and psychiatric comorbidity. An in-office induction allows a person to be professionally assessed for withdrawals and then monitored by trained staff to determine further dosing. ${ }^{29,30}$ The common daily dose for buprenorphine ranges from $2 \mathrm{mg}$ to $24 \mathrm{mg}$ daily, with the most frequently effective dose being between 12-20 mg daily. A frequently prescribed initiation dose is $4 \mathrm{mg}$ once in moderate withdrawals, with the option of taking another $4 \mathrm{mg}$ if needed for a total of $8 \mathrm{mg}$ on the first day. ${ }^{31}$ There is value in rapidly titrating the dose to prevent relapse back to more hazardous opioid use. Doses above 16-20mg have some diminishing additional benefit as the mu opioid receptors occupied do not increase dramatically beyond $16 \mathrm{mg}$. One significant benefit of buprenorphine's partial agonist mechanism is the resultant ceiling effect which significantly reduces the risk of overdose on buprenorphine. ${ }^{32}$ Typically 1-week supplies are given to start which allows for balance between flexibility and accountability. At follow up, lab testing is conducted typically with urine screening to both confirm the patient is taking the medication and to demonstrate cessation of the more problematic opioid. As patients report reduction in addiction related problems, then the buprenorphine prescriptions and lab monitoring can be extended out up to 30 days. ${ }^{31}$ Prescription durations are often gradually extended in a stepwise fashion from 1 week to 2 weeks to 3 weeks to 4 weeks to allow for more frequent safety monitoring early in treatment. The extent of screening process and frequency of monitoring should be weighed against the value of removing barriers to treatment in order to reduce relapses and overdoses on more harmful opioids. ${ }^{33}$ One of the primary leverage points when working with buprenorphine is the duration of prescriptions. Subsequently, when there are acute safety concerns or significant non-adherence to treatment recommendations, then the duration of the prescription can be shortened until the prescriber determines it is appropriate to extend again. This is commonly done when the lab testing shows high risk substance use in combination with the buprenorphine such as benzodiazepines, alcohol, methamphetamine, or highpotency opioids such as fentanyl. When it comes to tapering, patients and prescribers often desire to taper off more quickly than what the available evidence indicates is safe. Initially, providers should focus on establishing a consistent maintenance dose that establishes sobriety. Studies looking to evaluate the ideal duration of medication treatment with buprenorphine have consistently found that longer maintenance periods improve outcomes. ${ }^{34}$ In some countries, such as Norway, the approach to buprenorphine treatment of OUD is a lifelong recommendation. ${ }^{35}$ So at the onset, a useful approach is to plan for "as long as needed" which can be reassessed and individualized over time. Individuals that have opted to transition off buprenorphine due to side effects, should be offered naltrexone or methadone.

\section{Naltrexone}

A second widely available and frequently prescribed medication for OUD is long-acting injectable naltrexone. Naltrexone is a mu opioid receptor antagonist which is started orally once daily and if tolerated then is converted to the long acting injectable once monthly form. This treatment is sometimes preferable for individuals who are determined to completely avoid treatment with opioid agonists like buprenorphine and methadone. This medication does not lead to the physiologic dependence developed on opioid agonists like buprenorphine and methadone. However, in order to start naltrexone an individual must go through supported withdrawal in order to avoid a precipitated withdrawal. Oral initiation of naltrexone confirms tolerability and then the recommendation is conversion to the long acting injectable which has better evidence of efficacy for preventing relapse back to problematic opioid use. 
The evidence shows that once patients are through the more difficult induction process and have established regular use then the benefits of long-acting injectable naltrexone are comparable to buprenorphine treatment. Naltrexone is not a good option for individuals who are prescribed any opioid medications or for those who might be expected to be prescribed opioids in the foreseeable future. ${ }^{36,37}$

\section{Methadone}

A third commonly utilized medication for OUD is methadone. Depending on the country and regulatory environment in which a practitioner is working, methadone may be restricted to specialized clinics. Methadone is a full mu agonist with a long duration of action. As a full agonist, methadone has increased risk of over sedation and overdose. Additionally, methadone carries an increased risk of cardiac arrhythmias and medication interactions. With buprenorphine's more favorable safety profile, methadone is now often reserved for individuals for whom buprenorphine does not work. However, it is worth recognizing that methadone has been utilized since the 1960's with clearly demonstrated success in treating severe opioid use disorders. Like buprenorphine, the medical and social stability provided by methadone in reducing problematic opioid use and related behaviors significantly improves overall health and wellbeing. When methadone is prescribed, it is started with low doses of $10-30 \mathrm{mg}$ and increased gradually with close monitoring. The effective dose range for methadone is around $80-120 \mathrm{mg}$ daily. While methadone is sometimes used for short-term tapers as a part of medically managed withdrawal protocols, outcomes are better with longer durations of maintenance treatment. ${ }^{38-41}$

\section{Psychotherapy}

In parallel to consideration of appropriate medication treatment of OUD, there are multiple evidence-based psychotherapy approaches to recommend including cognitive behavioral therapy (CBT), motivational enhancement therapy (MET), contingency management (CM), and 12-step facilitation. CBT is the most widely researched form of psychotherapy and its systematic evaluation of thoughts, feelings, and behaviors has been successfully applied to support relapse prevention. Motivational enhancement therapy is the manualized form of motivational interviewing's collaborative, empathic approach to supporting individuals' own desire to change. Contingency management effectively incentivizes sobriety by providing rewards for appropriate urine drug screen results. 12-step facilitation is built on the 12 steps of alcoholics anonymous (AA) and centers around active peer support and a spiritual process. There are of course many additional forms of individual and group-based psychotherapy to support recovery from OUD. Ultimately, it is helpful to have a basic working knowledge of some of these approaches in order to both incorporate aspects into clinical encounters and to make informed referrals for continued treatment. ${ }^{42-44}$

\section{Community Support}

An important element of the therapeutic approach to OUD is the recommendation for patients to engage with community support. This is a somewhat unique paradigm of recovery from the disease of addiction; however, it is not all that different from the support groups that exist for other chronic diseases. There are robust community-based resources including peer-support meetings such as narcotics anonymous, alcoholics anonymous, smart recovery, and celebrate recovery. All of these are now increasingly available in online formats. ${ }^{2,45-47}$

Another important element of recovery from OUD is therapeutic housing, which includes "sober living" or "half-way" houses and apartments. Many people in recovery from OUD find this type of housing to be a valuable tool for additional accountability and social support.

Finally, most local laws subject people who struggle with OUD to criminalization for possession with subsequent social and financial consequences. Legal burdens and criminalization of people already struggling with addiction can worsen outcomes. In some places, people arrested for possessing or using controlled substances are sentenced instead to treatment courts which are also known as "drug" courts. This approach incentivizes sobriety and treatment engagement with the goal of rehabilitation and recovery rather than incarceration. ${ }^{48}$

\section{Special Populations}

There are a handful of particularly challenging OUD patient presentations ideally referred to an addiction specialist. These include pregnant patients, adolescents, patients with chronic pain and patients on MAT requiring acute pain relief. ${ }^{30}$ In pregnancy, methadone has been the mainstay for several decades but more recently buprenorphine has shown significant promise, whereas naltrexone or detoxification of pregnant women with OUD is not recommended. $^{49}$ In adolescents, medications are recommended for severe OUD; however, the evidence is quite limited. $^{50}$ In the setting of co-occurring chronic pain, 
methadone and buprenorphine may be preferable to naltrexone but even naltrexone is a viable option in the right circumstances. ${ }^{51}$ Finally, for patients requiring surgery or acute pain relief while on MAT, there are several options including temporary discontinuation, lowering or continuing the medication. Once again, this circumstance requires close coordination with an expert in addiction treatment and the other providers participating in the care. ${ }^{52}$

\section{Conclusion}

Ultimately, people who struggle with addiction to opioids suffer deeply from direct and indirect consequences of use. These individuals often come from difficult circumstances prior to developing an OUD, with various social, medical and psychiatric challenges. Subsequently, the suffering in these individuals' lives deeply impacts the families and communities around them. The privilege to care for OUD patients rests on the shoulders of all medical professionals. Thus, strengthening our knowledge of this illness and its treatments is critical. When aware of the disease and the ways to approach it, we can compassionately make progress in relieving at least a portion of this suffering.

\section{Disclosure}

The authors report no conflicts of interest in this work.

\section{References}

1. Brady K, Levin F, Galanter M, Kleber H. The American Psychiatric Publishing Textbook of Substance Use Disorder Treatment. 6th ed. American Psychiatric Publishing; 2021.

2. Strang J, Volkow ND, Degenhardt L, et al. Opioid use disorder. Nat Rev Dis Primer. 2020;6(1):3. doi:10.1038/s41572-019-0137-5

3. Kampman K, Jarvis M. American society of addiction medicine (ASAM) national practice guideline for the use of medications in the treatment of addiction involving opioid use. J Addict Med. 2015;9 (5):358-367. doi:10.1097/ADM.0000000000000166

4. Miller W, Rollnick S. Motivational Interviewing: Helping People Change. 3rd ed. Guilford Press; 2013.

5. Agerwala SM, McCance-Katz EF. Integrating screening, brief intervention, and referral to treatment (SBIRT) into clinical practice settings: a brief review. J Psychoactive Drugs. 2012;44(4):307-317. doi:10.1080/02791072.2012.720169

6. Blanco C, Volkow ND. Management of opioid use disorder in the USA: present status and future directions. Lancet. 2019;393 (10182):1760-1772. doi:10.1016/S0140-6736(18)33078-2

7. Compton WM, Valentino RJ, DuPont RL. Polysubstance use in the U.S. opioid crisis. Mol Psychiatry. 2021;26(1):41-50. doi:10.1038/ s41380-020-00949-3

8. Volkow ND, Jones EB, Einstein EB, Wargo EM. Prevention and treatment of opioid misuse and addiction: a review. JAMA Psychiatry. 2019;76(2):208-216. doi:10.1001/ jamapsychiatry.2018.3126

9. American Psychiatric Association. Diagnostic and Statistical Manual of Mental Disorders (DSM-5). 5th ed. American Psychiatric Association; 2013.
10. Mee-Lee D. The ASAM Criteria: Treatment Criteria for Addictive, Substance-Related, and Co-Occuring Conditions. 3rd ed. 2013.

11. Olsen A, Dwyer R, Lenton S. Take-home naloxone in Australia and beyond. Drug Alcohol Rev. 2018;37(4):437-439. doi:10.1111/ dar. 12700

12. Buresh M, Gicquelais RE, Astemborski J, Kirk GD, Mehta SH, Genberg BL. Fatal overdose prevention and experience with naloxone: a Cross-Sectional Study from a community-based cohort of people who inject drugs in Baltimore, Maryland. PLoS One. 2020;15(3):e0230127. doi:10.1371/journal.pone.0230127

13. Kerensky T, Walley AY. Opioid overdose prevention and naloxone rescue kits: what we know and what we don't know. Addict Sci Clin Pract. 2017;12(1):4. doi:10.1186/s13722-016-0068-3

14. Clark AK, Wilder CM, Winstanley EL. A systematic review of community opioid overdose prevention and naloxone distribution programs. $J$ Addict Med. 2014;8(3):153-163. doi:10.1097/ ADM.0000000000000034

15. Kosten TR, Baxter LE. Review article: effective management of opioid withdrawal symptoms: a gateway to opioid dependence treatment. Am J Addict. 2019;28(2):55-62. doi:10.1111/ajad.12862

16. Sigmon SC, Bisaga A, Nunes EV, O'Connor PG, Kosten T, Woody G. Opioid detoxification and naltrexone induction strategies: recommendations for clinical practice. Am J Drug Alcohol Abuse. 2012;38(3):187-199. doi:10.3109/00952990.2011.653426

17. Jones CM, McCance-Katz EF. Co-occurring substance use and mental disorders among adults with opioid use disorder. Drug Alcohol Depend. 2019;197:78-82. doi:10.1016/j.drugalcdep.2018.12.030

18. Donroe JH, Bhatraju EP, Tsui JI, Edelman EJ. Identification and management of opioid use disorder in primary care: an update. Curr Psychiatry Rep. 2020;22(5):23. doi:10.1007/s11920-02001149-0

19. World Health Organization. Guidelines for the psychosocially assisted pharmacological treatment of opioid dependence. World Health Organization. Available from: https://www.who.int/sub stance_abuse/publications/9789241547543/en/. Accessed March 28, 2021.

20. Shulman M, Wai JM, Nunes EV. Buprenorphine treatment for opioid use disorder: an overview. CNS Drugs. 2019;33(6):567-580. doi:10.1007/s40263-019-00637-z

21. Bentzley BS, Barth KS, Back SE, Book SW. Discontinuation of buprenorphine maintenance therapy: perspectives and outcomes. J Subst Abuse Treat. 2015;52:48-57. doi:10.1016/j.jsat.2014.12.011

22. Fiellin DA, Schottenfeld RS, Cutter CJ, Moore BA, Barry DT, O'Connor PG. Primary care-based buprenorphine taper vs maintenance therapy for prescription opioid dependence. JAMA Intern Med. 2014;174(12):1947-1954. doi:10.1001/jamainternmed.2014.5302

23. Johnson RE, Strain EC, Amass L. Buprenorphine: how to use it right. Drug Alcohol Depend. 2003;70(2 Suppl):S59-77. doi:10.1016/s03768716(03)00060-7

24. TIP 63: medications for opioid use disorder - full document | SAMHSA publications and digital products. Available from: https:// store.samhsa.gov/product/TIP-63-Medications-for-Opioid-UseDisorder-Full-Document/PEP20-02-01-006. Accessed March 28, 2021.

25. Lee JD, Grossman E, DiRocco D, Gourevitch MN. Home buprenorphine/naloxone induction in primary care. J Gen Intern Med. 2009;24 (2):226-232. doi:10.1007/s11606-008-0866-8

26. Cunningham CO, Giovanniello A, Li X, Kunins HV, Roose RJ, Sohler NL. A comparison of buprenorphine induction strategies: patient-centered home-based inductions versus standard-of-care office-based inductions. J Subst Abuse Treat. 2011;40(4):349-356. doi:10.1016/j.jsat.2010.12.002

27. Gunderson EW, Wang X-Q, Fiellin DA, Bryan B, Levin FR. Unobserved versus observed office buprenorphine/naloxone induction: a pilot randomized clinical trial. Addict Behav. 2010;35 (5):537-540. doi:10.1016/j.addbeh.2010.01.001 
28. Lee JD, Vocci F, Fiellin DA. Unobserved "home" induction onto buprenorphine. J Addict Med. 2014;8(5):299-308. doi:10.1097/ ADM.0000000000000059

29. Oesterle TS, Kolla B, Risma CJ, et al. Substance use disorders and telehealth in the COVID-19 pandemic era: a new outlook. Mayo Clin Proc. 2020;95(12):2709-2718. doi:10.1016/j.mayocp.2020.10.011

30. Oesterle TS, Thusius NJ, Rummans TA, Gold MS. Medicationassisted treatment for opioid-use disorder. Mayo Clin Proc. 2019;94 (10):2072-2086. doi:10.1016/j.mayocp.2019.03.029

31. Clinical guidelines for the use of buprenorphine in the treatment of opioid addiction: Treatment Improvement Protocol (TIP) series, No. 40; 2004. Available from: https://www.ncbi.nlm.nih.gov/books/ NBK64245/. Accessed May 27, 2021.

32. Coe MA, Lofwall MR, Walsh SL. Buprenorphine pharmacology review: update on transmucosal and long-acting formulations. J Addict Med. 2019;13(2):93-103. doi:10.1097/ ADM.0000000000000457

33. Jakubowski A, Fox A. Defining low-threshold buprenorphine treatment. $J$ Addict Med. 2020;14(2):95-98. doi:10.1097/ ADM.0000000000000555

34. Samples H, Williams AR, Crystal S, Olfson M. Impact of long-term buprenorphine treatment on adverse health care outcomes in medicaid. Health Aff. 2020;39(5):747-755. doi:10.1377/ hlthaff.2019.01085

35. Dalsbø TK, Steiro A, Strømme H, Reinar LM. Effectiveness of Tapering from Methadone or Buprenorphine Maintenance Treatment Compared to Traditional Maintenance Treatment for People with Opiate Addiction: Systematic Review. Norwegian Institute of Public Health; 2017.

36. Lee JD, Nunes EV, Novo P, et al. Comparative effectiveness of extended-release naltrexone versus buprenorphine-naloxone for opioid relapse prevention (X:BOT): a multicentre, open-label, randomised controlled trial. Lancet. 2018;391(10118):309-318. doi:10.1016/S0140-6736(17)32812-X

37. Jarvis BP, Holtyn AF, Subramaniam S, et al. Extended-release injectable naltrexone for opioid use disorder: a systematic review. Addict. 2018;113(7):1188-1209. doi:10.1111/add.14180

38. Joseph H, Stancliff S, Langrod J. Methadone maintenance treatment (MMT): a review of historical and clinical issues. Mt Sinai J Med. 2000;67(5-6):347-364.

39. Mattick RP, Breen C, Kimber J, Davoli M. Methadone maintenance therapy versus no opioid replacement therapy for opioid dependence. Cochrane Database Syst Rev. 2003;2:CD002209. doi:10.1002/ 14651858.CD002209

40. Johansson BA, Berglund M, Lindgren A. Efficacy of maintenance treatment with methadone for opioid dependence: a meta-analytical study. Nord J Psychiatry. 2007;61(4):288-295. doi:10.1080/ 08039480701415251
41. Wakeman SE, Larochelle MR, Ameli O, et al. Comparative effectiveness of different treatment pathways for opioid use disorder. JAMA Netw Open. 2020;3(2):e1920622. doi:10.1001/ jamanetworkopen.2019.20622

42. Dugosh K, Abraham A, Seymour B, et al. Review on the use of psychosocial interventions in conjunction with medications for the treatment of opioid addiction. J Addict Med. 2016;10(2):91-101. doi:10.1097/ADM.0000000000000193

43. Carroll KM, Onken LS. Behavioral therapies for drug abuse. Am $J \quad$ Psychiatry. 2005;162(8):1452-1460. doi:10.1176/appi. ajp.162.8.1452

44. Dutra L, Stathopoulou G, Basden SL, Leyro TM, Powers MB, Otto MW. A meta-analytic review of psychosocial interventions for substance use disorders. Am J Psychiatry. 2008;165(2):179-187. doi:10.1176/appi.ajp.2007.06111851

45. White WL. Addiction recovery mutual aid groups: an enduring international phenomenon. Addict. 2004;99(5):532-538. doi:10.1111/j.1360-0443.2004.00684.x

46. Galanter M, White WL, Hunter BD. Cross-cultural applicability of the 12-step model: a comparison of narcotics anonymous in the USA and Iran. $J$ Addict Med. 2019;13(6):493-499. doi:10.1097/ ADM.0000000000000526

47. Kelly JF. Is alcoholics anonymous religious, spiritual, neither? Findings from 25 years of mechanisms of behavior change research. Addict. 2017;112(6):929-936. doi:10.1111/add.13590

48. Joudrey PJ, Howell BA, Nyhan K, et al. Reporting of substance use treatment quality in United States adult drug courts. Int J Drug Policy. 2020;90:103050. doi:10.1016/j.drugpo.2020.103050

49. Tran TH, Griffin BL, Stone RH, Vest KM, Todd TJ. Methadone, buprenorphine, and naltrexone for the treatment of opioid use disorder in pregnant women. Pharmacotherapy. 2017;37(7):824-839. doi:10.1002/phar. 1958

50. Minozzi S, Amato L, Bellisario C, Davoli M. Maintenance treatments for opiate -dependent adolescents. Cochrane Database Syst Rev. 2014;(6):CD007210. doi:10.1002/14651858.CD007210.pub3

51. Oesterle TS, Kolla BP, Rummans TA, Gold MS. Medication-assisted therapies for opioid use disorders in patients with chronic pain. J Neurol Sci. 2020;411:116728. doi:10.1016/j.jns.2020.116728

52. Harrison TK, Kornfeld H, Aggarwal AK, Lembke A. Perioperative considerations for the patient with opioid use disorder on buprenorphine, methadone, or naltrexone maintenance therapy. Anesthesiol Clin. 2018;36(3):345-359. doi:10.1016/j.anclin.2018.04.002
International Journal of General Medicine

\section{Publish your work in this journal}

The International Journal of General Medicine is an international, peer-reviewed open-access journal that focuses on general and internal medicine, pathogenesis, epidemiology, diagnosis, monitoring and treatment protocols. The journal is characterized by the rapid reporting of reviews, original research and clinical studies across all disease areas. The manuscript management system is completely online and includes a very quick and fair peer-review system, which is all easy to use. Visit http://www.dovepress.com/ testimonials.php to read real quotes from published authors. 